Cahiers $d u$ MONDE RUSSE

\section{Cahiers du monde russe}

Russie - Empire russe - Union soviétique et États indépendants

$48 / 4 \mid 2007$

Varia

\title{
Adeeb Khalid, Islam after Communism
}

\section{Stéphane A. Dudoignon}

\section{OpenEdition \\ Journals}

Édition électronique

URL : https://journals.openedition.org/monderusse/6115

DOI : 10.4000/monderusse. 6115

ISSN : $1777-5388$

Éditeur

Éditions de l'EHESS

Édition imprimée

Date de publication : 2 décembre 2007

Pagination : 801-806

ISBN : 978-2-7132-2148-4

ISSN : $1252-6576$

\section{Référence électronique}

Stéphane A. Dudoignon, «Adeeb Khalid, Islam after Communism », Cahiers du monde russe [En ligne], 48/4 | 2007, mis en ligne le 16 juin 2009, consulté le 03 septembre 2022. URL : http://

journals.openedition.org/monderusse/6115; DOI : https://doi.org/10.4000/monderusse.6115

Ce document a été généré automatiquement le 3 septembre 2022.

Tous droits réservés 


\title{
Adeeb Khalid, Islam after Communism
}

\author{
Stéphane A. Dudoignon
}

\section{RÉFÉRENCE}

Adeeb KHALID, Islam after Communism. Religion and Politics in Central Asia.

Berkeley : University of California Press, 2007, 241 p., cartes, tabl., bibliogr., index

1 L'islam a-t-il été « dé-modernisé » (demodernized) en Asie centrale soviétique, comme l'auteur le suggère au cœur de son ouvrage (p. 115) - reprenant à son compte le concept de «re-traditionalisation» lancé au milieu des années 1990, à propos de l'islam centrasiatique, par Olivier Roy ? C'est en grande partie pour répondre à cette question récurrente qu'Adeeb Khalid, auquel on doit notamment une étude de référence sur l'islam réformé au début du $\mathrm{xx}^{\mathrm{e}}$ siècle (The Politics of Muslim Cultural Reform : Jadidism in Central Asia, chez le même éditeur, 1998), s'est attaqué, dix ans après ce premier et brillant essai, à la très ambitieuse synthèse dont il va être ici question. Axée sur le postulat que le poids de l'histoire importe - « history does matter» (p. 2) - dans tout effort de compréhension de l'islam moderne et contemporain en Asie centrale, cet ouvrage fait une place significative aux évolutions sur la longue et la moyenne durées, contrastant avec la vision purement synchronique adoptée par la très grande majorité des travaux récents sur le sujet. Dénonçant aussi la vision orientaliste encore dominante, qui postule l'existence d'un islam essentiel, invariant et transhistorique, fondé sur les écritures, l'auteur souligne à la fois la diversité interne de l'islam et le rôle qu'ont joué des domaines non strictement religieux, comme celui de la tradition éthique (adab), dans la transmission régionale de cette religion à travers les âges.

2 Ayant évoqué l'impact des mouvements messianiques $d u x^{\mathrm{e}}{ }^{\mathrm{e}}$ au $\mathrm{XV}^{\mathrm{e}}$ siècle, puis l'institutionnalisation des voies soufies sous la forme des modernesțarīāt, Adeeb Khalid met en lumière le paradoxe du quiétisme développé par la tradition juridique hanafite, conjugué au rôle politique souvent déterminant joué par les oulémas (comme dans le 
gouvernement de Tachkent, tout au long d'un très mouvementé xvIII siècle). Dans le même esprit, l'importance du legs mongol jusqu'à l'aube de l'époque moderne est à la fois confirmée et nuancée par l'incessante quête de légitimité islamique des émirs Manghit de Boukhara (1753-1920) dépourvus, eux, d'ascendance gengiskhanide. L'apport du monde nomade est, malheureusement, vite esquissé, à travers la prégnance de l'accès à l'islam à travers le lien avec les lignées sacrées : c'est bien peu, dans la mesure où la Transoxiane ellemême (région de peuplement majoritairement sédentaire située au nord de l'Amou-Daria), reste autant que la steppe profondément marquée par la dichotomie entre mondes sédentaire et nomade - comme en attestent, par exemple, le destin politique des Ouzbeks Laqay tout au long du $\mathrm{Xx}^{\mathrm{e}}$ siècle dans les régions méridionales de l'ancienne RSS des Tadjiks, leur rôle dans la résistance armée des années 1917-1929 et leur allégeance à la Naqshbandiyya leur ayant valu de se voir stigmatisés successivement et par le régime soviétique et par le pouvoir actuel au Tadjikistan. Cette négligence suggère que l'auteur, s'il vitupère avec vigueur et succès plus d'un stéréotype sur les sociétés centrasiatiques, en avalise quelques autres, à commencer par le caractère nécessairement superficiel de l'islamisation des nomades d'Asie centrale, véhiculé par la littérature ethnographique des périodes tsariste et soviétique, mais fortement remis en cause par les travaux d'éminents historiens actuels (sur les Kazakhs, par exemple, presque absents du présent ouvrage : Devin DeWeese, Allen J. Frank, Bruce G. Privratsky). La définition restrictive du concept d'Asie centrale apparaît du reste dès la carte $n^{\circ} 1$ : le domaine de la steppe y fait figure de tache blanche, l'actuel Xinjiang est ignoré, l'Afghanistan de même : c'est de l'Ouzbékistan, et dans une mesure moindre du Tadjikistan, qu'il est ici question, l'auteur avalisant une définition essentiellement péjorative de l'islam turkmène, kazakh et kirghiz.

S'étendant sur l'Empire russe et les enjeux de la modernité, l'auteur met l'accent sur l'ancienneté de la présence de populations musulmanes majoritaires dans le domaine des tsars (depuis l'intégration de la Haute-Volga à la Moscovie, à l'extrême fin du xIV siècle) et la grande diversité dans le temps et dans l'espace des réponses de l'État russe à ce défi démographique et culturel. Il nous rappelle, notamment, la mise en œuvre d'une politique d'isolement des institutions de l'islam par le général gouverneur Kaufman dans le territoire du Turkestan, à partir des années 1860. La réaction vernaculaire à la conquête russe est évoquée à travers les soulèvements successifs de la fin de la période tsariste (à Jizzakh sous la houlette des oulémas locaux en 1866, à Andidjan sous la direction de Dukhchi Ishan en 1898, etc.), mais aussi à travers le renforcement du pouvoir des émirs à Boukhara et une efflorescence sans précédent des constructions de madrasas par le capital musulman privé. Seconde lacune importante de l'ouvrage (récurrente, celle-là, chez l'auteur) : le réformisme islamique n'est évoqué que par le renouveau du début du $\mathrm{xx}^{\mathrm{e}}$ siècle (" djadidisme »), sans intérêt pour la documentation abondante - souvent manuscrite, il est vrai, et de langue persane -, du siècle précédent, laquelle a pourtant fait l'objet d'assez nombreuses publications dans les très nombreuses langues lues par l'auteur (l'allemand, en particulier). Historien distingué du djadidisme en Asie centrale et grand lecteur de Roger Chartier, Adeeb Khalid insiste sur le changement de la relation à l'autorité religieuse induit par l'essor moderne des techniques de communication, de l'imprimerie en particulier - tout en suggérant ailleurs que les violentes répressions des années 1930 et 1960 ont pu limiter la portée d'un tel phénomène. Par contre, le voile est à peine levé sur les réactions - ou l'absence de réactions - musulmanes à l'essor du djadidisme pendant la décennie qui suit la révolution de 1905, en dehors d'allusions à la période révolutionnaire et aux soulèvements de 1916 contre la colonisation russe des terres agricoles ou de pacage et la conscription militaire. 
Les premiers changements substantiels de la période soviétique sont mis en lumière par l'impact des campagnes antireligieuses, par l'effet de la création d'identités nationales séculaires, ainsi que par le rôle de nouvelles élites nationales dans la défense des valeurs associées à ces identités. Le récit commence avec la défaite des djadids par leurs opposants conservateurs dans les scrutins municipaux de l'été et de l'automne 1917, ce qui les conduisit sur la voie de la radicalisation. Se détournant de leur fascination initiale pour la culture libérale de l'Europe occidentale, ils adoptent une critique anticoloniale de l'ordre bourgeois (qu'illustrent par exemple les écrits antibritanniques de Firat en 1919 et en 1920) - confirmant la césure introduite par la Première Guerre mondiale dans la relation des mondes de l'islam à l'hégémonie des puissances chrétiennes, ce changement revêtant en Asie centrale des formes littéraires particulièrement précoces et vives, à la faveur de l'évaporation de la censure impériale, présente jusqu'à des dates plus tardives dans les empires britannique, français ou néerlandais. La différence est bien saisie, pour la période révolutionnaire, entre la République populaire des Conseils de Boukhara (1920-1924), où les djadids au pouvoir se rapprochent du mouvement nationaliste turc, et la République soviétique autonome du Turkestan, où la scène politique est dominée par des intellectuels et notables musulmans dotés d'une éducation russe - le plus souvent des Kazakhs originaires de la région du Semireche, « des hommes à l'aise avec le russe et avec les intrigues du pouvoir, et sans enracinement dans le réformisme islamique» (p.58). L'auteur restitue les oscillations de la politique bolchevique à l'encontre de l'islam, selon les circonstances générales, avec la création de "bureaux de la charia »(maḥkama-yi šar iyya)en 1922; le lancement de l'indigénisation des cadres du parti (korenizacija) en 1923 - entraînant la création d'une classe politique autochtone liée au régime soviétique; les conflits entre djadids et bolcheviks après les premiers assauts contre le mode de vie traditionnel en 1926 (l'activité religieuse publique étant limitée à partir de 1929); les changements substantiels induits par l'invasion nazie en 1941, les paysans d'Asie centrale conquérant au sein de l'Armée rouge leur statut de citoyens soviétiques (p.77), tandis qu'une Direction spirituelle des musulmans d'Asie médiane voit le jour en 1943 ; le retour des campagnes antireligieuses d'abord sous Hruščev, en 1959-1962, puis pendant la perestroïka...

5 L'auteur évoque en particulier, après les répressions qui commencent en 1927, le démantèlement des réseaux personnels des enseignants de madrasas et des maîtres des "voies» mystiques, porteurs de l'enseignement islamique classique, tandis que la fermeture des maktab-s (écoles coraniques) ne s'accompagne pas de l'apparition d'écoles soviétiques en nombre équivalent. Dès que le pouvoir soviétique parvient à contrôler les frontières de l'URSS, au tout début des années 1930, une forme nouvelle d'isolement se substitue à l'ouverture qu'avaient connue non seulement les djadids, comme le dit l'auteur, mais tous les courants de pensée, notamment mystiques, de la période présoviétique. Adeeb Khalid suggère que le " triomphe de la loi coutumière » a ramené l'islam centrasiatique à un état antérieur à l'émergence du mouvement djadid, tandis que la tradition islamique elle-même se voyait appauvrie par la répression. Pour autant, et c'est là une autre erreur d'appréciation substantielle de l'auteur, qui trop souvent adopte la position d'observateur lointain, la famille n'est pas devenue le «seul lieu » de transmission de l'islam : malgré l'incontestable saignée des répressions, les " causeries » (gaps-s)à contenu religieux, les «cellules " (hujra-s) clandestines et hôtelleries soufies ont perduré dans toute la Transoxiane d'un bout à l'autre du $\mathrm{xx}^{\mathrm{e}}$ siècle. Si des changements considérables sont indéniablement intervenus dans la pratique quotidienne de l'islam, un personnel religieux a continué d'officier, dont l'histoire n'est pas vraiment 
abordée ici, même si l'auteur a parfaitement perçu et bien restitué la permanence, dans l'esprit de la réforme islamique, du renouveau du commentaire coranique (tafsir)et du Hadith dans les madrasas tant officielles que clandestines d'Asie centrale, du début à la fin $\mathrm{du} \mathrm{xx}^{\mathrm{e}}$ siècle. Mais il est vrai que l'Ouzbékistan, considéré comme métonymie de l'Asie centrale dans son entier par une majorité de spécialistes de l'islam centrasiatique, et sur lequel l'auteur concentre son propos comme si cela allait de soi, ne constitue peutêtre pas le terrain le plus intéressant pour une étude historique de l'islam moderne et de son personnel religieux, du fait de son régime policier passé et présent, et des entraves croissantes mises à l'accès des chercheurs étrangers à de nombreuses archives publiques et privées.

6 Éludant ces difficultés de méthode, l'ouvrage se concentre sur les dernières décennies. Mettant en lumière le rôle de marqueur d'identité ethnique et de culture nationale graduellement dévolu à l'islam dans le cadre des anciennes républiques fédérées, Adeeb Khalid évoque avec justesse le contrat social entre un État soviétique dispensateur de stabilité, de niveau de vie et de prestige international, et des populations centrasiatiques qui intègrent progressivement, sans toujours se renier, les normes de sociabilité nouvellement édictées à leur attention. Un exemple : l'adaptation d'une institution comme les maitresses enseignant l'éthique traditionnelle (otin-s) en Transoxiane, nanties d'une nouvelle légitimité, entrainnant la féminisation de certaines fonctions et sociabilités religieuses - ce qui fera beaucoup craindre ces femmes savantes par les pouvoirs actuels en Ouzbékistan et au Tadjikistan -, n'est pas sans rappeler la place prise par les lettrées musulmanes turcophones (ābiztāy-s - de l'arabe ḥāfiž, récitant du Coran) de la MoyenneVolga dans la lutte contre les campagnes de christianisation entre le début du XVIII ${ }^{e}$ siècle et le début du $\mathrm{xx}^{\mathrm{e}}$ (voir sur ce point les travaux d'Agnès Kefeli). Sacrifiant parfois à quelques stéréotypes à la vie dure, notamment de l'intelligentsia russe sur l'Asie centrale, l'auteur présente la région comme "dépourvue de dissidents et de samizdat», nonobstant la richesse, aujourd'hui documentée, de l'underground islamique sous Brežnev et ses successeurs - voir le rôle de ces libraires et éditeurs tadjiks tel Mullā Ǐsāân Jān Mullā Muḥammad Hwāja (né en 1943) venant de Douchanbe à Tachkent, à partir du milieu des années 1970, imprimer leurs ouvrages didactiques, la nuit, sur les presses des Éditions d'État de la RSS ouzbèke (témoignage oral recueilli par l'auteur de ces lignes auprès de l'intéressé en juillet 2004). Inversement, dans le but de prendre le contrepied de lieux communs véhiculés par maints écrits occidentaux, Adeeb Khalid ne fait parfois que verser dans un excès contraire. Ainsi lorsqu'il affirme que, jusqu'à la fin de la période soviétique, l'islam non officiel est resté "apolitique». Certes la tradition quiétiste transmise par le rite hanafite a continué d'exercer son influence - les notions de « confiance en Dieu » (tawakkul) et de « patience » (Sabr) étaient au centre de l'enseignement théologique dispensé pendant des décennies à Douchanbe par le célèbre « Mawlawī Hindūstānī » (1892-1989) -, cependant elle n'a pas tardé à trouver ses limites, avec le «schisme» des principaux disciples ferghanais de Hindūstān, reprochant à ce dernier son apolitisme (voir, sur ce phénomène, les nombreux travaux de Bahtiar Babadžanov, pourtant mentionnés dans l'ouvrage), et l'émergence de courants ouvertement hostiles au « clergé » officiel comme le Mouvement pour le renouveau islamique de la jeunesse au Tadjikistan, qui se constitue dans les kolkhozes cotonniers de la région de Qurghan-Teppa à partir du milieu des années 1970. Si la réislamisation « par le bas » que l'on a observée à partir de la fin des années 1980 a bénéficié de l'appui d'organisations multinationales apolitiques (dans la mesure où elles ne s'intéressent pas, ès-qualité, à la prise du pouvoir, tels le Tablīgī Jamā’at pakistanais, 
ou les Nurcu turcs et le réseau d'écoles de Fethullah Gülen évoqués par l'auteur dans son chapitre 5), il est par contre tout simplement naïf de dénigrer toute dimension politique à l'activité de ces dernières, ou de prétendre qu'un pays comme l'Iran ne joue pas de rôle dans les processus en cours, au prétexte de l'incompatibilité doctrinale de sa majorité chiite dja farite avec le sunnisme hanafite qui domine en Asie centrale. Dans ce dernier cas, par exemple, l'auteur n'a pas du tout été sensible au rôle prépondérant, depuis la fin $\mathrm{du} \mathrm{xx}^{\mathrm{e}}$ siècle, des grandes madrasas sunnites du Khorasan et du Baloutchistan iraniens, lesquelles drainent chaque année des centaines d'étudiants en religion du Tadjikistan et s'efforcent d'attirer, par une politique de communication agressive, les futurs imams et muftis des autres États d'Asie centrale, voire du Caucase et de Russie d'Europe. Pour ne rien dire du nombre certes très limité encore, mais sans cesse croissant des étudiants tadjiks qui, délaissant le sunnisme de leurs pères, s'en vont quérir des diplômes de théologie chiite à la grande madrasa "Imam-Khomeyni » de Qom, avant de revenir ouvrir chez eux ce que l'on appelle déjà, au Tadjikistan, les mosquées «iraniennes». Les uns comme les autres, sunnites et néo-chiites, arrivent pour leurs études en Iran, nantis de visas en bonne et due forme, ce qui dénote bien la volonté de la République islamique, là comme ailleurs, de développer sur le moyen terme une politique de puissance régionale de premier plan, en jouant comme de coutume sur une pluralité de tableaux. Plus largement, l'auteur est parfaitement fondé à affirmer, dans ses ultimes chapitres sur la situation actuelle, que de nombreux djihads contemporains de l'ancienne aire soviétique (Afghanistan et Tchétchénie, en particulier) ne peuvent être expliqués par la seule référence à l'islam - les facteurs régionaux devant être davantage pris en considération. Pour autant, ces derniers sont évoqués de manière souvent très abstraite. Par ailleurs, il n'est pas justifié de dire que les thèmes soulevés par la propagande du parti islamiste multinational Hizb al-Tahrir n'ont que peu de résonance en Asie centrale, où ce dernier gagne au contraire en audience dans la jeunesse estudiantine et les classes moyennes inférieures, contribuant à miner le prestige plus que jamais chancelant des autorités traditionnelles de l'islam, compromises aux yeux des masses populaires par leur association avec des régimes perçus localement comme autant de "kleptocraties " appuyées sans vergogne par les puissances occidentales. C'est là que réside, au demeurant, le vrai défi pour les décennies à venir: le remplacement déjà en cours d'un personnel religieux formé localement pendant la période soviétique par une typologie beaucoup plus large de jeunes clercs - très souvent fils des précédents - éduqués depuis une décennie à l'étranger (Iran, Pakistan, Égypte, notamment), mais aussi d'intellectuels et d'activistes politiques de l'islam, rompus aux techniques de communication électronique, conduisant à une forme de re-modernisation en accéléré, qu'a déjà commencé de manifester la multiplication, en Asie centrale, des conflits internes à la communauté des croyants.

8 Au total, c'est un essai exceptionnellement bien écrit, dans un anglais littéraire d'une très grande clarté, que nous offre Adeeb Khalid. Nous faisant partager sa grande expérience de l'enseignement et de la communication de la recherche, l'auteur donne une leçon d'écriture pour laquelle son ouvrage devrait être proposé en modèle aux générations futures de spécialistes. Au-delà de ces considérations de forme, son livre brille d'une grande indépendance d'esprit, marquée par de nombreuses et parfois audacieuses innovations conceptuelles. La moindre n'est pas un effort constant de mise en contexte, notamment politique, mais aussi économique et social, encore bien rare pourtant chez les spécialistes de l'islam centrasiatique. Comme l'auteur le postule justement, à plusieurs 
reprises, l'islam de l'Asie centrale actuelle ne peut être compris sans qu'il soit tenu compte de l'impact spécifique de la période soviétique, même si cet impact reste très largement à évaluer, et à étudierles permanences qui ont aussi pu se manifester dans un grand nombre de niches institutionnelles, sociales ou géographiques - avec l'aval des polices politiques de l'URSS (comme le révèlent des documents du KGB sur la mobilisation par ce dernier des grandes figures du « souterrain» islamique pendant l'occupation de l'Afghanistan). Il est dommage, en particulier, que ce très riche sous-terrain, où se situe l'essentiel du matériau brut de la période soviétique, n'ait pas fait ici l'objet d'un début d'exploration, celle-ci nécessitant un très important, aléatoire et épuisant travail d'histoire orale dans les villes et les campagnes, quartier par quartier, village par village. Du moins la publication du présent ouvrage, ses échos critiques immédiats et divers attestent-ils de l'importance stratégique aujourd'hui reconnue à l'islam centrasiatique -importance excessive, du reste, témoignant de la promotion univoque, par la science occidentale, de la révolution religieuse comme horizon politique unique des sociétés non européennes depuis la révolution iranienne de 1978-1979, montrant aussi notre paresse à interroger l'histoire sociale et culturelle globale autrement plus complexe de l'Asie centrale, une région demeurée l'une des dernières véritables terrae incognitae de l'histoire contemporaine. 